\title{
Protein expression status of p53 and epidermal growth factor receptor in thymoma
}

\author{
FEI CUI ${ }^{1}$, JIANXING $\mathrm{HE}^{2}$, JUN LIU ${ }^{2}$ and YIJIANG CHEN ${ }^{1}$ \\ ${ }^{1}$ Department of Thoracic and Cardiovascular Surgery, the First Affiliated Hospital of Nanjing Medical University, Nanjing; \\ ${ }^{2}$ Department of Thoracic Surgery, the First Affiliated Hospital of Guangzhou Medical College, Guangzhou, P.R. China
}

Received September 9, 2010; Accepted December 27, 2010

DOI: 110.3892/ol.2011.267

\begin{abstract}
Evaluating the state of malignancy of thymoma is a challenge due to the discrepancies between pathological classifications and clinical stage criteria. Therefore, the identification of markers contributing to the assessment of the stage of malignancy of thymoma would be useful. This study aimed to evaluate the prevalence of p53 and epidermal growth factor receptor (EGFR) in thymoma, demonstrate the clinical significance and assess the potential of specifictargeted therapy. A total of 16 thymic hyperplasia patients and 43 thymoma patients were included in the study. Samples were tested for the expression of p53 and EGFR using immunohistochemistry. In the multivariate analysis, the thymoma cases were followed up to analyze the relationship between survival rates and a number of potential factors, such as p53 and EGFR. There were 21 completely resected stage II cases which were evaluated for relapse-free time. The distribution of p53 with clinicopathological parameters was: type A/AB $57.1 \%$, type B1 $85.7 \%$, type B2 $85.7 \%$, type B3 $90.9 \%$ and type C $100 \%$; and stage I $90.9 \%$, stage II $90.9 \%$, stage III $100 \%$ and stage IV $100 \%$. The EGFR-positive rates were: type A/AB $42.9 \%$, type B1 71.4\%, type B2 $57.1 \%$, type B3 $90.9 \%$ and type C $100 \%$; and stage I $45.5 \%$, stage II $76 \%$, stage III $75 \%$ and stage IV $100 \%$. EGFR expression correlated with tumor size, pathological classification and advanced clinical stage, whereas p53 correlated only with pathological classifications. Findings of our multivariate analysis showed that neither p53 nor EGFR are independent prognostic factors. Nevertheless, the fact that a statistical difference $(p<0.05)$ was noted in relapse-free survival time between the EGFRpositive and EGFR-negative groups $(48.182 \pm 33.757$ vs. $76.3 \pm 10.339$ months) suggests that EGFR plays a key role in thymoma progression. No positive results were found between
\end{abstract}

Correspondence to: Professor Yijiang Chen, the First Affiliated Hospital of Nanjing Medical University, No. 300 Guangzhou Avenue, Nanjing, Jiangsu 0086-210029, P.R. China

E-mail: yijiangchen2009@hotmail.com

Key words: p53, epidermal growth factor receptor, thymoma, clinicopathological parameters, survival the p53 groups following a survey conducted to assess relapse time. Therefore, the application of EGFR-targeting agents is warranted in invasive thymoma, whereas the targeting of p53 has yet to be elucidated.

\section{Introduction}

Thymoma is a rare anterior mediastinal tumor with complicated processing mechanisms. The pathological classifications were based on the WHO classification system. Thymomas were classified into types A, B and C (1). On the other hand, the current clinical diagnosis is predominantly based on the estimate of surgical management established by Masaoka et al (2). However, pathologically indolent cases occasionally show malignancy as invasion, recurrence or metastasis (3). For this reason, discrepancies are noted between clinical stages and the current pathological classifications. Therefore, certain markers should be defined as a complement of clinicopathological parameters to evaluate malignancy and outcome. Surgical resection is the optimal standard therapy. Based on the largesample analysis of 1,320 cases in Japan, surgical treatment was shown to be an independent factor affecting prognosis, but the value of adjuvant chemotherapy and radiotherapy is uncertain (4). However, in terms of the unresectable or relapsed cases, a non-surgical therapeutic approach is the last recourse. Thus, alternative therapeutic molecular targets are required.

As a key indicator, mutant p53 is predisposed to cancer (5). Certain authors studied the accumulation of an abnormal form of the protein in the cell nucleus, the normal p53 protein with a half-life that was too short to be detected, while most, but not all, mutant p53 proteins possess a prolonged half-life, accumulate in tissues, and are able to be directly detected by immunohistochemical assays (6,7). To exclude false-positive and false-negative results, the analysis was performed a number of times according to the most frequently used method, i.e., immunohistochemistry with certain specific antibodies, such as DO-7. p53 protein accumulation is interpreted as being indicative of the presence of p53 mutations. As such, the immunohistochemical evaluation of p53 revealed its prognostic significance in the tumor $(8,9)$.

Epidermal growth factor receptor (EGFR), a cellular surface membrane glycoprotein receptor, plays a key role in the regulation of key normal cellular processes and in the hyperproliferation, infiltration, invasion and metastasis of 
epithelium-derived tumors. Thus, gene and biological therapies targeting EGFR were extensively applied to the treatment of certain solid tumors $(10,11)$.

Studies previously reported p53 and EGFR in thymoma (12-20). However, the majority of these studies lack a control group with benign thymic tissues or a long-term follow-up study due to low morbidity. In this study, the expression of p53 and EGFR in thymoma was tested using an immunohistochemical assay. Correlations between p53 and EGFR and clinicopathological characteristics were analyzed and the survival rate was investigated.

\section{Materials and methods}

Patients and samples. A total of 16 thymic hyperplasia and 43 thymoma patients were recruited in the First Affiliated Hospital of Guangzhou Medical College and the First Affiliated Hospital of Nanjing Medical University, China. The patients were pathologically diagnosed between 2002 and 2005, and the 43 thymoma patients were available for a 5-year follow-up study. Standards were adhered to according to the ? Ethics Committee, and written informed consent was obtained from the patients. In the thymoma patients, ages ranged from 12-72 years (mean 45.16), with 27 females and 16 males (Table I). The pathological classification was in accordance with the WHO 1999 pathological classification criteria. The distribution was: 2 with type A, 5 with type $\mathrm{AB}, 7$ with type B1, 14 with type B2, 11 with type $\mathrm{B} 3$ and 4 with type $\mathrm{C}$. The clinical staging was based on Masaoka's staging system as follows: 11 patients in stage I, 25 in stage II, 4 in stage III and 3 in stage IV (Table II).

Immunohistochemical assay. The protein expression of EGFR and p53 were evaluated immunohistochemically in representative paraffin-embedded sections. The staining was performed with mouse monoclonal antibody (clones EGFR 111.6, and p53 DO-7), purchased from Thermo Fisher Scientific, Inc. (Waltham, MA, USA). The procedure was performed in accordance with the manual's instructions. The diaminobenzidine (DAB) immunohistochemistry kit with antimouse secondary antibody (Invitrogen, USA) was used to visualize the antibody binding. The p53-positive expression was considered to be the nuclear staining, while the EGFR expression was assessed visually as indicating the tumor cell membrane, cytoplasm or both. p53 scores were assigned: 0 , negative; $1+, 10 \%$ nuclei staining positively; $2+, 10-50 \%$ nuclei; $3+,>50 \%$ nuclei (21). The EGFR-positive expression was scored as $0,1+, 2+$ and $3+$ in accordance with Henley's system (15).

Follow-up study. A follow-up study was conducted for the thymoma cases that were diagnosed between 2002 and 2005. Six patients were lost at follow-up and 3 patients succumbed to diseases of other systems. A total of 43 cases were available for the 5-year survival, and the overall survival was defined as the time from diagnosis to succumbing to disease. A total of 21 stage II cases, who had undergone a complete resection without adjuvant chemo- or radiotherapy, were selected to calculate the relapse-free time. The relapse-free time was defined as the period of time from surgery until relapse or progress. The patients were either contacted through routine outpatient services or telephone interviews were conducted.

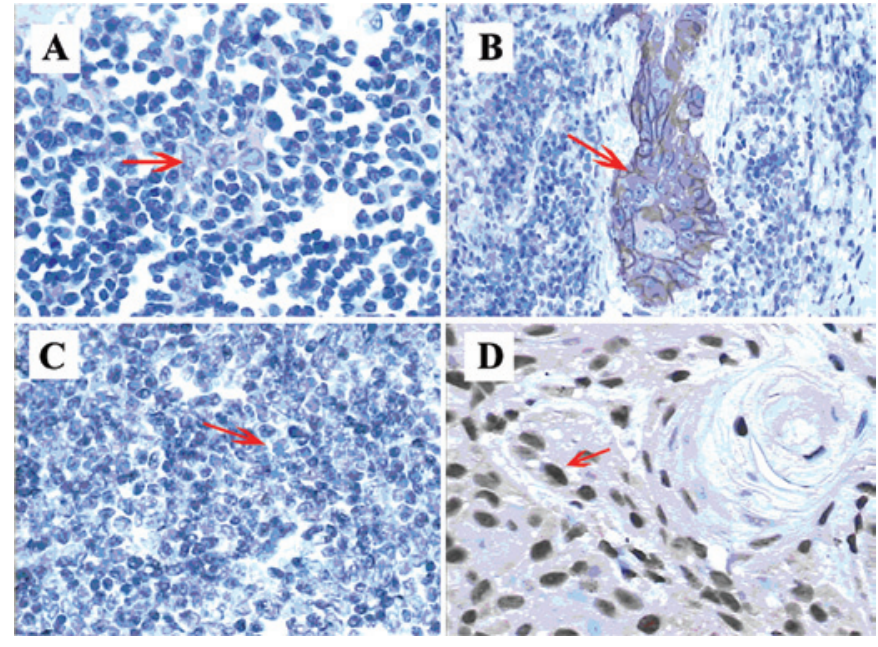

Figure 1. Epidermal growth factor receptor (EGFR) and p53 expression in thymoma. (A) EGFR weak expression in thymoma epithelial cells: a faint beige staining of cytoplasm was observed and the final score was $1+$. (B) EGFR strong-positive expression in malignant thymoma: the membrane and cytoplasm were stained brown showing strong staining. Thus, the final score was 3+. (C) p53-negative in thymoma: no nuclear staining was observed in the tumor cells. (D) p53 strong-positive in thymoma: the nuclear staining was dark brown and $>50 \%$ of the cells were positive. As a result, the final score was $3+$.

The follow-up time was $48.326 \pm 21.646$ months in the overall survival study.

Statistical analysis. The data were analyzed by software SPSS 17.0. The Chi-square test was used to compare the variations between the two groups. The Spearman's model was performed to analyze the correlation coefficient. Follow-up was obtained in 43 cases and was terminated in August 2010. A number of factors, including pathological classification, clinical stage, EGFR expression, p53 expression and completed resection, were considered for the multivariate analysis. The multivariate analysis, conducted with the application of Cox regression, was used to evaluate the effect of the selected prognostic factors on survival. Relapse-free curves were analyzed by the Kaplan-Meier method and compared using the log-rank test. $\mathrm{P}<0.05$ was considered to be statistically significant.

\section{Results}

Fig. 1 shows the expression of EGFR and p53 in immunohistochemical assays.

Patient characteristics are shown in Table I. Neither p53 nor EGFR correlated with age, gender or myasthenia gravis morbidity $(\mathrm{p}>0.05)$. Table I also shows the $\mathrm{p} 53$-positive rates (56.3 vs. $86 \%, \mathrm{p}<0.05)$ in thymic hyperplasia and thymoma; the EGFR-positive rate was 18 and $70 \%$, respectively $(\mathrm{p}<0.05)$. The results of thymic hyperplasia and thymoma were significantly different from those of EGFR. However, EGFR was found to correlate with tumor size, whereas p53 did not.

p53 expression. Table II shows the distribution of p53 expression with WHO 1999 classification in 64 patients: type A/AB $57.1 \%$, type B1 $85.7 \%$, type B2 $85.7 \%$, type B3 $90.9 \%$ and type $\mathrm{C} 100 \%(\mathrm{p}<0.05, \mathrm{r}=0.471)$. The positive rates of $\mathrm{p} 53$ 
Table I. The clinicopathological characteristics of epidermal growth factor receptor (EGFR) and p53.

\begin{tabular}{|c|c|c|c|c|c|c|}
\hline & \multicolumn{3}{|c|}{ EGFR } & \multicolumn{3}{|c|}{ p53 } \\
\hline & Positive (\%) & Negative $(\%)$ & p-value & Positive (\%) & Negative $(\%)$ & p-value \\
\hline Age (years) & & & 1.000 & & & 1.000 \\
\hline$<50$ & $17(68.0)$ & $8(32.0)$ & & $21(84.0)$ & $4(16.0)$ & \\
\hline$\geq 50$ & $13(72.2)$ & $5(27.2)$ & & $15(83.3)$ & $3(16.7)$ & \\
\hline Gender & & & 0.307 & & & 0.229 \\
\hline Male & $13(81.3)$ & $3(18.7)$ & & $15(93.4)$ & $1(6.6)$ & \\
\hline Female & $17(63.0)$ & $10(37.0)$ & & $21(77.8)$ & $6(22.2)$ & \\
\hline Myasthenia gravis & & & 0.727 & & & 0.652 \\
\hline Positive & $9(75.0)$ & $3(25.0)$ & & $11(91.6)$ & $1(8.4)$ & \\
\hline Negative & $21(67.7)$ & $10(22.3)$ & & $25(80.7)$ & $6(29.3)$ & \\
\hline Tumor size (cm) & & & 0.011 & & & 0.082 \\
\hline$<6$ & $8(47.1)$ & $9(53.9)$ & & $11(68.8)$ & $5(31.2)$ & \\
\hline$\geq 6$ & $22(84.6)$ & $4(15.4)$ & & $25(92.6)$ & $2(7.4)$ & \\
\hline Histology & & & 0.001 & & & 0.030 \\
\hline Thymoma & $30(70.0)$ & $13(30.0)$ & & $37(86.0)$ & $6(14.0)$ & \\
\hline Thymus hyperplasia & $3(18.8)$ & $13(81.2)$ & & $9(56.3)$ & $7(43.7)$ & \\
\hline
\end{tabular}

Table II. Epidermal growth factor receptor (EGFR) and p53 with pathological classifications and clinical stages.

\begin{tabular}{|c|c|c|c|c|c|c|c|c|c|c|}
\hline & \multicolumn{5}{|c|}{ EGFR } & \multicolumn{5}{|c|}{ p53 } \\
\hline & 0 & $1+$ & $2+$ & $3+$ & Statistical value & 0 & $1+$ & $2+$ & $3+$ & Statistical value \\
\hline Pathological & & & & & $\mathrm{p}<0.001$ & & & & & $\mathrm{p}<0.001$ \\
\hline classification & & & & & $\mathrm{r}=0.515$ & & & & & $\mathrm{r}=0.471$ \\
\hline A & 1 & 1 & 0 & 0 & & 1 & 1 & 0 & 0 & \\
\hline $\mathrm{AB}$ & 3 & 2 & 0 & 0 & & 2 & 2 & 1 & 0 & \\
\hline B1 & 2 & 2 & 3 & 0 & & 1 & 6 & 0 & 0 & \\
\hline $\mathrm{B} 2$ & 6 & 2 & 3 & 3 & & 2 & 4 & 5 & 3 & \\
\hline B3 & 1 & 1 & 5 & 4 & & 1 & 4 & 3 & 3 & \\
\hline $\mathrm{C}$ & 0 & 1 & 1 & 2 & & 0 & 1 & 1 & 2 & \\
\hline Total & 13 & 9 & 12 & 9 & & 7 & 18 & 10 & 8 & \\
\hline Clinical stage & & & & & $\begin{array}{l}\mathrm{p}=0.003 \\
\mathrm{r}=0.443\end{array}$ & & & & & $\begin{array}{c}\mathrm{p}=0.289 \\
\mathrm{~N} / \mathrm{A}\end{array}$ \\
\hline I & 6 & 4 & 0 & 1 & & 1 & 6 & 4 & 0 & \\
\hline II & 6 & 5 & 9 & 5 & & 6 & 9 & 4 & 6 & \\
\hline III & 1 & 0 & 1 & 2 & & 0 & 2 & 2 & 0 & \\
\hline IV & 0 & 0 & 2 & 1 & & 0 & 1 & 0 & 2 & \\
\hline Total & 13 & 9 & 12 & 9 & & 7 & 18 & 10 & 8 & \\
\hline
\end{tabular}

expression in the various clinical stages were analyzed (stage I $90.9 \%$, stage II $90.9 \%$, stage III $100 \%$ and stage IV $100 \%, \mathrm{p}=0.289>0.05)$. As a result, no significance was noted between positive and negative groups in p53 expression.

Epidermal growth factor receptor expression. Table II shows the positive distribution of thymoma pathological types. The positive rates vs. pathological types were: $42.9 \%$ for type $\mathrm{A} / \mathrm{AB}$, $71.4 \%$ for type B1, $57.1 \%$ for type B2, $90.9 \%$ for type B3 and $100 \%$ for type $\mathrm{C}$. The positive rates in the various types of thymoma were significantly different $(\mathrm{p}<0.05, \mathrm{r}=0.515)$.
Regarding the rank correlation between the positive rates and different clinical stages (stage I $45.5 \%$, stage II $76 \%$, stage III $75 \%$ and stage IV 100\%), a statistical significance was noted among the respective stages $(\mathrm{p}<0.05)$ with a moderate relativity $(r=0.443)$.

Follow-up results. Table III shows the results of the multivariate analysis with a variety of factors, including pathological classification, clinical stage, p53 expression, EGFR expression, completed resection, tumor size (cutoff $6 \mathrm{~cm}$ ) and myasthenia gravis. The Cox regression model showed that only clinical 
Table III. Multivariate analysis of several potential factors with overall survival (43 cases).

\begin{tabular}{lccc}
\hline & No. of cases & Survival (\%) & p-value \\
\hline $\begin{array}{l}\text { Pathological } \\
\text { classification }\end{array}$ & & & 0.072 \\
A & 2 & $2(100.0)$ & \\
AB & 5 & $5(100.0)$ & \\
B1 & 7 & $5(71.4)$ & \\
B2 & 14 & $12(85.7)$ & \\
B3 & 11 & $7(63.6)$ & \\
C & 4 & $1(25.0)$ & \\
Clinical stage & & & 0.048 \\
I & 11 & $11(100.0)$ & \\
II & 25 & $20(80.0)$ & \\
III & 4 & $2(50.0)$ & \\
IV & 3 & $0(0.0)$ & \\
EGFR expression & & & 0.933 \\
Positive & 30 & $21(70.0)$ & \\
Negative & 13 & $12(92.3)$ & \\
p53 expression & & & 0.128 \\
Positive & 36 & $27(75.0)$ & \\
Negative & 7 & $6(85.7)$ & \\
Completed resection & & & 0.678 \\
Yes & 38 & $32(84.2)$ & \\
No & 5 & $1(20.0)$ & \\
Tumor size (cm) & & & 0.954 \\
$<6$ & 17 & $16(94.1)$ & \\
$\geq 6$ & 26 & $17(65.4)$ & \\
Myasthenia gravis & & & 0.437 \\
Positive & 12 & $10(83.3)$ & \\
Negative & 31 & $23(74.2)$ & \\
\hline
\end{tabular}

EGFR, epidermal growth factor receptor.

stage significantly affected overall survival $(\mathrm{p}=0.048<0.05)$ among the potential factors. Neither $\mathrm{p} 53(\mathrm{p}=0.128>0.05)$ nor EGFR ( $\mathrm{p}=0.933>0.05)$ was shown to be an independent prognostic factor.

In the 21 radically resected cases with stage II, a significant difference was noted in the relapse-free survival time between the EGFR-negative and EGFR-positive groups $(76.3 \pm 10.339$ vs. $48.182 \pm 33.757$ months, $\mathrm{p}=0.039<0.05$ ). No relapse was noted in the negative group, but a recurrence was noted in 4 cases in the positive group within 1 year. No difference was noted between the p53-positive and p53-negative groups $(74.2 \pm 8.677$ vs. $79.667 \pm 13.997$ months, $\mathrm{p}=0.942>0.05$ ). The relapse-free curves are shown in Fig. 2.

\section{Discussion}

Thymoma is a controversial issue. Previous studies have shown the prognostic significances of pathological classification, clinical stage and completed resection (4,22-24). Nevertheless, the results were inconsistent due to the rare incidence and complicated mechanisms of thymoma. For

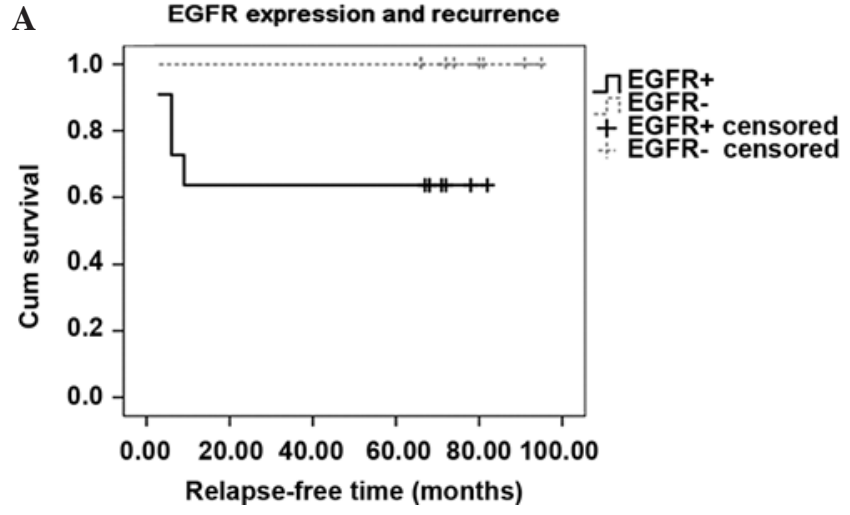

B

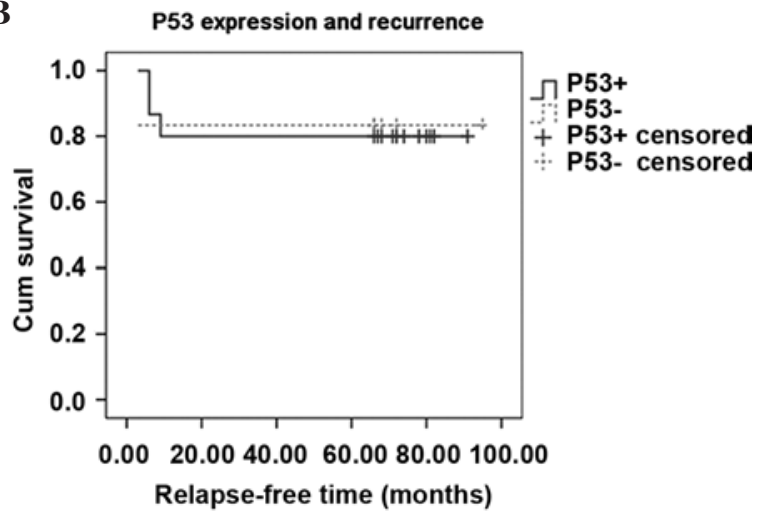

Figure 2. Correlation of p53 and epidermal growth factor receptor (EGFR) expression with relapse-free survival in 21 stage II patients (11 EGFR-positive vs. 10 EGFR-negative cases) that had undergone a completed resection. The follow-up survey was completed in July 2010. (A) There were 4 recurrences in the EGFR-positive cases, but no recurrence was noted in the negative group. A statistical difference $(\mathrm{p}<0.05)$ was noted between the positive (solid line) and the negative cases (dashed line). (B) Relapse-free survival between the two groups (16 p53-positive vs. 5 p53-negative cases) is shown. One relapse was noted in the p53-negative cases (dashed line) and three relapses occurred in the p53-positive cases (solid line), indicating no significant difference $(\mathrm{p}>0.05)$

example, our present study showed that only clinical stage is an independent prognostic factor, as compared to pathological classification and completed resection. In addition, although thymoma is basically indolent in its early stage, certain investigators asssert that occasionally benign-appearing thymoma is potentially malignant $(25,26)$. Furthermore, clinical doctors have not achieved a standardized solution to classify thymoma into typical TMN classifications. The above phenomena in thymoma makes it difficult to evaluate patient outcome. Therefore, numerous checkpoint proteins were examined in thymoma in order to prove their prognostic or predictive value, and their contribution to the outcome evaluation (20). However, few studies have investigated multiple factors in these tumors, due to the fact that they lacked a control group from normal thymoma tissues or the follow-up study had insufficient data.

The present study focused on the significance between p53 and EGFR, and clinicopathological characteristics and thymic hyperplasia. Furthermore, the potential factors, including pathological classification, clinical stage, p53 expression, EGFR expression, completed resection, tumor size and myasthenia gravis, were subject to a multivariate analysis. We found that p53 rarely correlated with the pathological classifications and differed in thymic hyperplasia and thymoma. 
Consequently, the value of protein accumulation in p53 is limited, since the protein accumulation did not accurately express the status of p53 mutation. Therefore, combining p53 genetic analysis with the immunohistochemical assay is required in order to clarify its significance in thymoma (27). The prevalence of EGFR is more valuable in thymoma in that it is well-correlated with the above pathological typing, clinical staging and tumor size and contributes to distinguishing between thymic hyperplasia and thymoma. The overexpression of EGFR in invasive thymoma was previously reported, but the significance of the survival rates was not determined. Although findings of the multivariate analysis did not confirm whether EGFR was an independent prognostic factor, the present follow-up data indicated that the completely resected cases with EGFR expression showed a significantly higher relapse rate than the negative group in stage II, suggesting that EGFR plays a key role in thymoma progression. Therefore, if the internal relationships between EGFR expression and thymoma and the accompanying mechanism are clarified, an approach may be developed that contributes to the evaluation of patient outcome with EGFR testing (ELISA and preoperative biopsy). Moreover, in the event that positive results are obtained, a specific therapy may be developed.

The role of surgery in thymoma is crucial. However, rapidly developing non-surgical approaches have currently become the major treatment for cases which are unresectable or in which relapse occurs. Since EGFR is much more valuable than $\mathrm{p} 53$, the former is considered a potential aim for targeted agents. Furthermore, various trials have been conducted on the application of EGFR-targeted agents in advanced thymoma cases $(28,29)$. A number of these trials showed a favorable response, indicating that these drugs can be applied for malignant thymoma. Notably, the results of our study did not reveal the clinical significance of p53 and EGFR in thymoma, but a rational approach for the application of targeted therapies. Subsequently, if the significances of EGFR-targeted agents are confirmed in invasive thymoma, particularly for pre-operative neo-adjuvant chemotherapy, tumor size may be reduced and complete resection or even survival rates may increase.

\section{Acknowledgements}

We thank Yingying Gu for the technical assistance on immunohistochemistry assay and Yan Zhong for the data collection.

\section{References}

1. Marx A and Muller-Hermelink H: From basic immunobiology to the upcoming WHO-classification of tumors of the thymus. The Second Conference on Biological and Clinical Aspects of Thymic Epithelial Tumors and related recent developments. Pathol Res Pract 195: 515-533, 1999.

2. Masaoka A, Monden Y, Nakahara K and Tanioka T: Follow-up study of thymomas with special reference to their clinical stages. Cancer 48: 2485-2492, 1981.

3. Suster S: Diagnosis of thymoma. J Clin Pathol 59: 1238-1244, 2006.

4. Kondo K and Monden Y: Therapy for thymic epithelial tumors: a clinical study of 1,320 patients from Japan. Ann Thorac Surg 76 878-884, 2003

5. Vousden KH: Outcomes of p53 activation - spoilt for choice. J Cell Sci 119: 5015-5020, 2006.

6. Tominaga O, Hamelin R, Remvikos Y, Salmon R and Thomas G: p53 from basic research to clinical applications. Crit Rev Oncog 3: 257-282, 1992.
7. Shiao YH, Palli D, Caporaso NE, et al: Genetic and immunohistochemical analyses of p53 independently predict regional metastasis of gastric cancers. Cancer Epidemiol Biomarkers Prev 9: 631-633, 2000

8. Steels E, Paesmans M, Berghmans T, et al: Role of p53 as a prognostic factor for survival in lung cancer: a systematic review of the literature with a meta-analysis. Eur Respir J 18: 705-719, 2001.

9. Pectasides D, Papaxoinis G, Nikolaou M, et al: Analysis of 7 immunohistochemical markers in male germ cell tumors demonstrates the prognostic significance of p53 and MIB-1. Anticancer Res 29: 4151-4156, 2009.

10. Carpenter G and Cohen S: Epidermal growth factor. J Biol Chem 265: 7709-7712, 1990 .

11. Fischer O, Hart S, Gschwind A and Ullrich A: EGFR signal transactivation in cancer cells. Biochem Soc Trans 31: 12031208,2003

12. Ionescu D, Sasatomi E, Cieply K, Nola M and Dacic S: Protein expression and gene amplification of epidermal growth factor receptor in thymomas. Cancer 103: 630-636, 2005.

13. Hayashi $\mathrm{Y}$, Ishii $\mathrm{N}$, Obayashi $\mathrm{C}$, et al: Thymoma: tumour type related to expression of epidermal growth factor (EGF), EGF-receptor, p53, v-erb B and ras p21. Virchows Arch 426: 43-50, 1995.

14. Sasaki H, Yukiue H, Sekimura A, et al: Elevated serum epidermal growth factor receptor level in stage IV thymoma. Surg Today 34: 477-479, 2004.

15. Henley J, Koukoulis G and Loehrer PL Sr: Epidermal growth factor receptor expression in invasive thymoma. J Cancer Res Clin Oncol: 167-170, 2002.

16. Aisner SC,Hameed MR, Wang W, et al: EGFR and C-Kit immunostaining in advanced or recurrent thymic epithelial neoplasms staged according to the WHO: an Eastern Cooperative Oncology Group Study. American Society of Clinical Oncology 22: 9637, 2004.

17. Girard N, Shen R, Guo T, et al: Comprehensive genomic analysis reveals clinically relevant molecular distinctions between thymic carcinomas and thymomas. Clin Cancer Res 15: 6790-6799, 2009.

18. Suzuki E, Sasaki H, Kawano O, et al: Expression and mutation statuses of epidermal growth factor receptor in thymic epithelial tumors. Jpn J Clin Oncol 36: 351-356, 2006.

19. Tomita M, Matsuzaki Y and Onitsuka T: Relationship between expression of cancer-related proteins and tumor invasiveness in thymoma. Eur J Cardiothorac Surg 21: 595-598, 2002.

20. Baldi A, Ambrogi V, Mineo D, et al: Analysis of cell cycle regulator proteins in encapsulated thymomas. Clin Cancer Res 11: 5078-5083, 2005.

21. Landolfi JA and Terio KA: Transitional cell carcinoma in fishing cats (Prionailurus viverrinus): pathology and expression of cyclooxygenase-1, -2 and p53. Vet Pathol 43: 674-681, 2006.

22. Detterbeck FC: Clinical value of the WHO classification system of thymoma. Ann Thorac Surg 81: 2328-2334, 2006.

23. Detterbeck FC and Parsons AM: Thymic tumors. Ann Thorac Surg 77: 1860-1869, 2004.

24. Yin H, Du J, Lu Z, Jiao X, Wang J and Zhou X: The correlation of the World Health Organization histologic classification of thymic epithelial tumors and its prognosis: a clinicopathologic study of 108 patients from China. Int J Surg Pathol 17: 255-261, 2009.

25. Riedel RF and Burfeind WR Jr: Thymoma: benign appearance, malignant potential. Oncologist 11: 887-894, 2006.

26. Reddy RHV, Shah R, Kumar B and Thorpe JAC: Recurrence of stage I thymoma in sternum, 13 years after 'complete' excision. Eur J Cardiothorac Surg 23: 134-135, 2003.

27. Shiao Y-H, Palli D, Caporaso NE, et al: Genetic and immunohistochemical analyses of p53 independently predict regional metastasis of gastric cancers. Cancer Epidemiol Biomarkers Prev 9: 631-633, 2000.

28. Bedano PM, Perkins S, Burns M, et al: A phase II trial of erlotinib plus bevacizumab in patients with recurrent thymoma or thymic carcinoma. American Society of Clinical Oncology 26: 19087, 2008.

29. Christodoulou C, Murray S, Dahabreh J, et al: Response of malignant thymoma to erlotinib. Ann Oncol 19: 1361-1362, 2006. 